\title{
A Synapse-Specific Carbohydrate at the Neuromuscular Junction: Association with Both Acetylcholinesterase and a Glycolipid
}

\author{
Leland J. C. Scott, Francis Bacou, and Joshua R. Sanes \\ Department of Anatomy and Neurobiology, Washington University School of Medicine, St. Louis, Missouri 63110
}

With the aim of investigating the roles of carbohydrates in synapse formation, we have characterized a synapse-specific saccharide at the vertebrate neuromuscular junction. Two lectins of similar specificity (Dolichos biflorus agglutinin, DBA, and Vicia villosa- $B_{4}$ agglutinin, VVA-B ${ }_{4}$ ) stain synaptic but not extrasynaptic regions of the rat muscle fiber surface and thus define a synapse-specific carbohydrate. Using these and other probes, we show that the carbohydrate moiety concentrated at the neuromuscular junction resembles $\mathrm{N}$-acetylgalactosamine (GaINAc) linked in the $\beta$-anomeric form to the termini of oligosaccharides. VVA-B $B_{4}$ also selectively stains neuromuscular junctions in human, mouse, rabbit, guinea pig, chick, frog, axolotl, snake, fish, and lamprey muscles, a phylogenetic conservatism that suggests a synapse-related role for GalNAc $\beta$-terminal saccharides.

AChE from muscle binds to DBA- and VVA-B ${ }_{4}$-agarose, and is thereby identified as a glycoconjugate bearing the synapse-specific carbohydrate. Assay of AChE isoforms reveals that asymmetric, collagen-tailed forms, known to be highly concentrated at the rat neuromuscular junction, bind DBA and VVA-B ${ }_{4}$, while globular forms, which are more widely distributed, do not. A second class of GalNAC-bearing glycoconjugates is demonstrable immunohistochemically with monoclonal antibodies to stage-specific embryonic antigen (SSEA)-3 (Shevinsky et al., 1982) and GM2 (Natoli et al., 1986), which recognize GalNAc-bearing glycolipids. These antibodies selectively stain neuromuscular junctions, where they recognize glycolipid-like molecules that bind VVA-B ${ }_{4}$ but are distinguishable from AChE. The association of a synapse-specific carbohydrate with at least 2 different synapse-specific molecules raises the possibility that the former plays a role in determining a property that the latter share, such as concentration at the synapse.

The carbohydrate components of glycoconjugates have long been thought to be involved in a variety of cell-cell interactions, and it has been hypothesized that this is true for synaptic interactions

\footnotetext{
Received June 16, 1987; revised Sept. 1, 1987; accepted Sept. 5, 1987.

We thank Dorothy Dill, Sue Eads, and Jeanette Cunningham for assistance; B. Caterson, J. Dodd, S. Hakamori, T. Jessell, P. Livingston, T. Rosenberry, and D. Solter for antibodies; S. Tollefson for VVA; and S. Kornfeld and D. Purves for comments. This work was supported by grants from the Muscular Dystrophy Association and the National Institutes of Health. L.J.C.S. was supported in part by NIH RSA GM07200 from the NIGMS. F.B.'s permanent address is Institut National de la Recherche Agronomique, Station Physiologie Animale, Montpellier, 34060, France.

Correspondence should be addressed to Joshua R. Sanes, Department of Anatomy and Neurobiology, Washington University School of Medicine, 660 South Euclid Avenue, Box 8108, St. Louis, MO 63110.

Copyright (C) 1988 Society for Neuroscience $0270-6474 / 88 / 030932-13 \$ 02.00 / 0$
}

as well (see, for example, Margolis and Margolis, 1978; Hakamori, 1981; Olden et al., 1982). We have previously shown that the lectin Dolichos biflorus (DBA) selectively stains neuromuscular junctions in rat skeletal muscle and thus defines a synapsespecific carbohydrate (Sanes and Cheney, 1982). This result has been confirmed in several laboratories (Hansen-Smith, 1984; Kaupmann et al., 1985; Askanas et al., 1986; Ribera et al., 1987). The existence of this synapse-specific carbohydrate and the relative simplicity of the neuromuscular junction make this an attractive system in which to study the involvement of carbohydrates in synapse formation and function. We have therefore begun to characterize the synaptic carbohydrates recognized by DBA. First, we used a panel of lectins, antibodies, and glycosidases of known specificities as histochemical probes to map the structure of the carbohydrate(s) concentrated at the rat neuromuscular junction. Second, we asked whether a similar carbohydrate is concentrated at skeletal neuromuscular junctions in other vertebrates. Finally, we used histochemical and biochemical methods to seek glycoconjugates with which the carbohydrate is associated. We report that the synapse-specific carbohydrate resembles a terminal, nonreducing $\beta$-linked $N$-acetylgalactosaminyl moiety, that it is concentrated at neuromuscular junctions in a variety of species, and that it is associated with at least 2 glycoconjugates: $\mathrm{AChE}$ and a glycolipid. The phylogenetic conservation of this carbohydrate and its association with more than a single synapse-specific molecule suggest that the carbohydrate plays a role in synapse formation or function.

Portions of this work have been presented in abstract form (Scott and Sanes, 1984, 1987).

\section{Materials and Methods}

Lectins and antibodies. Lectins used in this study are listed in Tables 1 and 2. Biotinylated Con A, lima bean lectin (LBL), Lens culinaris (LCA), peanut agglutinin (PNA), Psophocarpus tetragonobulus agglutinin (PTA), Ricinis communis agglutinin I and II (RCA, and $\mathrm{RCA}_{\mathrm{II}}$ ), soybean agglutinin (SBA), Ulex europeus agglutinin I (UEA-I), and WGA, as well as fluoresceinated BSA- $\mathrm{A}_{4}$, phytohemagglutinin $\mathrm{E}$ and $\mathrm{L}$ (PHA-E and PHA-L), and unconjugated Con $A$ and VVA-B $B_{4}$ were obtained from Sigma (St. Louis, MO). Biotin-Helix pomatia agglutinin (HPA) was from Vector (San Francisco, CA). Biotinylated DBA and LBL, and unconjugated Salvia sclerea agglutinin (SSA) were from E.Y. Laboratories (San Mateo, CA). VVA-A $A_{4}$ was a gift of S. Tollefsen (Washington University).

DBA was purified according to the method of Etzler and Kabat (1970), except that Synsorb A (Chembiomed, Edmonton, Alberta, Canada) was used instead of hog mucin-agarose as the affinity matrix. VVA-B $B_{4}$ was purified according to the method of Tollefsen and Kornfeld (1983), except that Synsorb 57 (Chembiomed) was used as the affinity matrix. Dolichos biflorus seeds were from F. W. Schumacher (Sandwich, MA), and Vicia villosa seeds were from Mangelsdorf (St. Louis, MO).

DBA, VVA-A , $_{4}$ VVA-B ${ }_{4}$, and SSA were biotinylated using $N$-biotinyl- 
Table 1. Staining of synaptic and extrasynaptic portions of rat skeletal muscle fiber surfaces by lectins with varying specificities

\begin{tabular}{|c|c|c|}
\hline Lectin & Nominal specificity $^{a}$ & $\begin{array}{l}\text { Synaptic (S) } \\
\text { and } \\
\text { extrasynaptic } \\
\text { (E) staining }\end{array}$ \\
\hline Ulex europeus agglutinin (UEA) & Fuc & None \\
\hline Concanavalin A (Con A) & Man, Glc & $S \cong E$ \\
\hline Lens culinaris (LCA) & Man, Glc & $S \cong E$ \\
\hline Wheat germ agglutinin (WGA) & NeuAc, GleNAc & $\mathrm{S} \cong \mathrm{E}$ \\
\hline Ricinis communis agglutinin I $\left(\mathrm{RCA}_{1}\right)$ & Gal & $S \cong E$ \\
\hline Ricinis communis agglutinin II $\left(\mathrm{RCA}_{\mathrm{II}}\right)$ & Gal, GalNAc & $S \cong E$ \\
\hline Phytohemagglutinin E (PHA-E) & Complex $^{c}$ & $S \cong E$ \\
\hline Phytohemagglutinin L (PHA-L) & Complex ${ }^{d}$ & $\mathbf{S} \cong \mathbf{E}$ \\
\hline Bandeiraea simplicifolia $\mathrm{A}_{4}\left(\mathrm{BSA}-\mathrm{A}_{4}\right)$ & Gal, GalNAc & $S \cong E$ \\
\hline Vicia villosa $\mathrm{A}_{4}$ isolectin (VVA-A ${ }_{4}$ ) & Gal, GalNAc & $\mathrm{S} \cong \mathrm{E}$ \\
\hline Peanut agglutinin (PNA) $)^{b}$ & $\mathrm{Gal} \beta(1-3)$ GalNAc & $\mathrm{S} \geq \mathrm{E}$ \\
\hline Helix pomatia agglutinin (HPA) & GalNAc, Gal & $\mathrm{S}>\mathrm{E}$ \\
\hline Soybean agglutinin (SBA) & GalNAc, Gal & $\mathrm{S}>\mathrm{E}$ \\
\hline Dolichos biflorus agglutinin (DBA) & GalNAc & $S \gg E$ \\
\hline Vicia villosa $\mathrm{B}_{4}$ isolectin (VVA-B $\mathrm{B}_{4}$ ) & GalNAc & $S \gg E$ \\
\hline
\end{tabular}

aan, mannose; Glc, glucose; Gal, galactose; Fuc, fucose; GlcNAc, $N$-acetylglucosamine; GalNAc, $N$-acetylgalactosamine; NeuAc, neuraminic (sialic) acid.

. PNA appears to stain presynaptic elements at the neuromuscular junction, as well as synaptic and extrasynaptic portions of the muscle fiber surface (see also Smith and Ko, 1986).

' Biantennary, galactosylated $N$-linked oligosaccharides with a bisecting $N$-acetylglucosamine on the $\beta$-linked mannose residue of the core (Cummings and Kornfeld, 1982).

${ }^{d}$ Tri- and tetraantennary glycopeptides, terminated in galactose or $N$-acetylgalactosamine, and having at least one of the $\alpha$-linked mannose residues substituted with a $\beta$-GlcNAc at C-2 and C-4 (Cummings and Konfeld, 1982).

$\omega$-aminocaproic acid $N$-hydroxysuccinimide ester (Enzotin; EnzoBiochem, NY). Lectins were dialyzed against 200 mM sodium bicarbonate buffer, $\mathrm{pH} 8.0$, and $100 \mathrm{~mm}$ GalNAc was added to protect binding sites. Enzotin was dissolved at $1.5 \mathrm{mg} / \mathrm{ml}$ in dimethylformamide, $-15^{\circ} \mathrm{C}$, and added to the lectin solution dropwise with stirring. Optimal lectin activity was obtained when the Enzotin : lectin ratio was $\sim 50: 1$. Derivatizations were carried out at room temperature for $4 \mathrm{hr}$, after which the lectins were desalted on Sephadex G-10.
DBA and VVA- $\mathrm{B}_{4}$ were conjugated at $1-2 \mathrm{mg}$ protein $/ \mathrm{ml}$ gel to Affigel 15 (Biorad) according to the manufacturer's directions. Coupling was at $\mathrm{pH} 7.8$ in $200 \mathrm{~mm}$ HEPES/bicarbonate buffer with $100 \mathrm{~mm}$ GalNAc. After $4 \mathrm{hr}$, unreacted gel was quenched with $100 \mathrm{~mm}$ ethanolamine, $\mathrm{pH}$ 8.0.

Monoclonal antibodies to SSEA-3 (Shevinsky et al., 1982) and SSEA-4 (Kannagi et al., 1983b) were provided by D. Solter (Wistar Institute, Philadelphia, PA) and also by J. Dodd and T. Jessell (Columbia Uni-

Table 2. Staining of synaptic and extrasynaptic portions of rat skeletal muscle fiber surfaces by lectins and antibodies that recognize GalNac

\begin{tabular}{|c|c|c|c|c|}
\hline \multirow[b]{2}{*}{ Probe } & \multicolumn{3}{|c|}{ Blood group specificity } & \multirow{2}{*}{$\begin{array}{l}\text { Synaptic } \\
\text { (S) and } \\
\text { extra- } \\
\text { synaptic } \\
\text { (E) staining }\end{array}$} \\
\hline & $\begin{array}{l}\text { In } \\
\text { (O-linked } \\
\text { GalNAc) }\end{array}$ & $\begin{array}{l}\text { A } \\
(\alpha \text {-GalNAc })\end{array}$ & $\begin{array}{l}\mathrm{Cad} / \mathrm{Sda} \\
(\beta \text {-GalNAc) }\end{array}$ & \\
\hline \multicolumn{5}{|l|}{ Lectins } \\
\hline Dolichos biflorus agglutinin (DBA) & + & + & + & $S \gg E$ \\
\hline Helix pomatia agglutinin (HPA) & + & + & + & $\mathrm{S}>\mathrm{E}$ \\
\hline Soy bean agglutinin (SBA) & + & + & + & $\mathrm{S}>\mathrm{E}$ \\
\hline Salvia sclerea agglutinin (SSA) & + & - & + & $\mathrm{S} \gg \mathrm{E}$ \\
\hline Vicia villosa $\mathrm{B}_{4}$ isolectin (VVA-B $\left.\mathrm{B}_{4}\right)$ & + & - & + & $\mathrm{S} \gg \mathrm{E}$ \\
\hline Lima bean lectin (LBL) & + & + & - & None $^{b}$ \\
\hline Psophocarpus tetragonolobus agglutinin (PTA) & + & + & - & None $^{b}$ \\
\hline \multicolumn{5}{|l|}{ Monoclonal antibodies } \\
\hline $\mathrm{CB}$ & + & + & - & None ${ }^{b}$ \\
\hline $\mathrm{Cul}$ & + & + & - & None $^{b}$ \\
\hline Lu81 & + & + & - & None $^{b}$ \\
\hline HH1 & $?$ & + & - & None $^{b}$ \\
\hline HH5 & $?$ & + & - & None ${ }^{L}$ \\
\hline $\mathrm{M} 1 / 22.25$ & - & $+^{a}$ & - & None $^{b}$ \\
\hline
\end{tabular}

${ }^{a}$ Recognized Forsmann antigen, GalNAc $\alpha 1-3 \mathrm{GalNAc} \beta 1-3 \mathrm{Gal} \beta-\mathrm{R}$.

${ }^{b}$ Shown to be active by staining of known GalNAc-rich control tissues (guinea pig ilium or rat kidney). 
versity, NY). Blood group A-specific monoclonal antibodies Lu8 1, Cu 1, HH1, and HH5 (Clausen et al., 1985; Hirohashi et al., 1985) were provided by S. I. Hakomori (University of Washington, Seattle, WA); CB (Hurakawa et al., 1985) was purchased from Immucor (Norcross, GA), anti-Forsmann antigen, M1/22.25 (Stern et al., 1978), was provided by T. Jessell and J. Dodd, and anti-GM2 (Natoli et al., 1986) was the gift of P. Livingston (Sloan-Kettering Cancer Center, New York). Antibodies to chondroitin 0-, 4-, and 6-sulfates (Couchman et al., 1984) were the gift of B. Caterson (University of West Virginia, Morgantown, WV). Rabbit antiserum to human erythrocyte AChE was the gift of T. Rosenberry (Case Western Reserve, Cleveland, $\mathrm{OH}$ ).

Histology. Cryostat sections of unfixed skeletal muscles were prepared and stained essentially as described by Covault and Sanes (1986). The sections were incubated first with biotinylated lectin (usually at 2-5 $\mu \mathrm{g}$ / $\mathrm{ml}$ ) or antibody and then with an appropriate fluorescein-conjugated reagent: streptavidin (Bethesda Research Laboratories, Gaithersburg, MD) or avidin (EY Laboratories) for biotinylated lectins, goat antirabbit IgG (Cappel Laboratories, Cochranville, PA) for antisera, and goat anti-mouse IgG or goat anti-mouse IgM (Cappel Laboratories or Jackson Immunoresearch, Avondale, PA) for monoclonal antibodies. Fluorescein-avidin was dissolved in carbonate-bicarbonate buffer, $\mathrm{pH}$ 8.7 , to reduce nonspecific binding of avidin; other incubations and all washes were in PBS. Rhodamine- $\alpha$-bungarotoxin, prepared as described in Ravdin and Axelrod (1977), was included in either the first or second incubation to mark synaptic sites. Stained sections were mounted in glycerol containing paraphenylenediamine, and viewed with fluorescein and rhodamine optics.

For glycosidase digestion, cryostat sections were incubated with 36 milliunits of $\alpha$-N-acetylhexosaminidase from C. lampas (Miles, Naperville, IL) or $\beta-N$-acetylhexosaminidase from jack bean (Sigma) in pH 4.0 citrate buffer for $5 \mathrm{hr}, 37^{\circ} \mathrm{C}$, or with 36 or 36,000 milliunits of $\beta$-galactosidase from $E$. coli (Sigma) for $30 \mathrm{~min}$ to $5 \mathrm{hr}$ at $37^{\circ} \mathrm{C}$. Sections to be stained with antibodies to glycosaminoglycans were treated with chondroitinase $\mathrm{ABC}$ as described by Couchman et al. (1984). For blocking studies, lectins were mixed with potential inhibitors before being applied to sections. These were: $N$-acetylgalactosamine, $\alpha$-methylmannoside, galactose, glucose, chondroitin sulfate from shark cartilage or bovine trachea, dermatan sulfate from porcine skin, heparin from bovine lung or porcine mucosa, mucin from porcine stomach (all from Sigma), heparan sulfate (Miles), or chemically desulfated chondroitin (prepared from chondroitin sulfate by the method of Danishefsky et al., 1960). For studies of glycolipids, sections were extracted with chloroform : methanol $(2: 1)$ at $-70^{\circ} \mathrm{C}$ for $15-45 \mathrm{~min}$, and then rinsed with saline. Treated sections were stained as described above.

For electron microscopy, a rat platysma muscle was incubated successively with biotinylated VVA-B ${ }_{4}$ and HRP-conjugated avidin (Cappel Laboratories), then fixed and reacted for peroxidase. Some bundles of fibers were teased from the muscles for light microscopy, while others were refixed in $\mathrm{OsO}_{4}$, dehydrated, and embedded in Araldite for thinsectioning (see Covault and Sanes, 1986, for details).

Extraction and assay of $A C h E$. To extract total AChE from muscle, endplate-rich regions (Hall, 1973) of rat diaphragm were homogenized in 4 volumes of a "high-salt" buffer $(1 \mathrm{M} \mathrm{NaCl}, 15 \mathrm{~mm}$ HEPES, $1 \%$ Triton X-100, $10 \mathrm{~mm}$ EGTA, $5 \mathrm{~mm} N$-ethylmaleimide, $2 \mathrm{~mm}$ benzamidine, $50 \mu \mathrm{g} / \mathrm{ml}$ leupeptin, $100 \mu \mathrm{g} / \mathrm{ml}$ aprotinin) and centrifuged at $40,000 \times \mathrm{g}, 4^{\circ} \mathrm{C}$ for $30 \mathrm{~min}$; the supernatant was retained. For sequential extraction of AChE forms, the method of Younkin et al. (1982) was used. Muscle was first homogenized in "low-salt" buffer ("high-salt" buffer without $\mathrm{NaCl}$ ) and centrifuged as above. The supernatant containing the globular $\mathrm{AChE}$ was removed, and the pellet was resuspended in high-salt buffer, incubated on ice for $60 \mathrm{~min}$, then centrifuged at $40,000 \times g$ for $60 \mathrm{~min}$. The resulting supernatant was enriched in asymmetric AChE. AChE was analyzed by velocity sedimentation on $5-20 \%$ sucrose gradients, as described by Hall (1973). AChE activity was assayed by the colorimetric method of Ellman et al. (1961) or by the radiometric method of Johnson and Russell (1975).

AChE binding to lectins was determined by incubating aliquots of muscle extract with lectin-conjugated agarose. Typically, tests used 100 $\mu \mathrm{l}$ of lectin gel in a total volume of $400 \mu \mathrm{l}$. Immediately after adding extract to the gel, the tubes were centrifuged briefly, and an aliquot of the supernatant was removed. The tubes were remixed and then incubated for $4-12 \mathrm{hr}$ at $4^{\circ} \mathrm{C}$ with gentle rocking, after which another aliquot of supernatant was removed. The 2 aliquots were assayed for AChE activity, and the difference between them was assumed to represent $\mathrm{AChE}$ bound to the gel. Control tubes containing unconjugated gel showed that inactivation of $\mathrm{AChE}$ during incubation and nonspecific binding of $\mathrm{AChE}$ to gel were insignificant.

\section{Results}

\section{Characterization of a synaptic carbohydrate}

Cryostat sections of rat diaphragm were incubated with one of a series of biotinylated lectins, then stained with fluoresceinavidin or -streptavidin and rhodamine- $\alpha$-bungarotoxin. $\alpha$-Bungarotoxin binds to acetylcholine receptors (AChRs) in the postsynaptic membrane and so marks synaptic sites. As previously reported (Sanes and Cheney, 1982), the lectin DBA stained synaptic sites intensely, but did not detectably stain extrasynaptic portions of muscle fibers or other intramuscular structures, such as blood vessels or nerve trunks (Fig. 1, $a, b$ ). DBA is known to recognize terminal GalNAc residues (Hammerstrom et al., 1977) and, as expected, its staining of neuromuscular junctions was blocked by $10 \mathrm{~mm}$ GalNAc, but not by other monosaccharides (glucose, $\alpha$-methyl mannoside, galactose, or $N$-acetylglucosamine, all at $100 \mathrm{~mm}$ ). HPA and SBA, lectins that are selective for terminal GalNAc but can also bind other saccharides (Pereira and Kabat, 1974; Hammerstrom et al., 1977; Baker et al., 1983), stained synaptic sites intensely, but also stained extrasynaptic areas faintly (Fig. $1, c, d)$. In contrast, several lectins that recognize other sugar moieties stained both synaptic and extrasynaptic portions of muscle fibers approximately equally (Fig. 1,e, f; Table 1). Together, these results show that while the entire muscle fiber surface is rich in carbohydrate, a terminal saccharide that contains or resembles GalNAc is highly concentrated at the neuromuscular junction.

There are 3 well-defined ways in which GalNAc can be linked to subterminal structures: (1) directly O-linked to serine or threonine residues of proteins, (2) as an $\alpha$-linked terminal sugar of an oligosaccharide, or (3) as a $\beta$-linked terminal sugar. These 3 configurations are found in the human blood group antigens Tn, A, and Cad (or Sda), respectively, and, as such, have been studied in detail (Cartron and Nurden, 1979; Donald et al., 1982; Blanchard et al., 1985; Furukawa et al., 1985; Hirohashi et al., 1985). Terminal GalNAc residues may also be linked to subterminal sugars by a phosphodiester bond (Balsamo et al., 1986; see also Marchase, 1987); however, this linkage has not been well characterized and we do not consider it here. DBA recognizes GalNAc in its $\mathrm{O}-, \alpha$-, and $\beta$-linked conformations and thus cannot be used to distinguish among them (Etzler et al., 1981; Blanchard et al., 1983). This is true for IIPA and SBA as well (Pereira and Kabat, 1974; Hammerstrom et al., 1977; Blanchard et al., 1983; Mansson and Olofsson, 1983). However, several lectins and monoclonal antibodies are available that can differentiate these structures. We used a battery of such reagents to determine the conformation of GalNAc at the neuromuscular junction.

The lectins PTA (Appukuttan and Basu, 1981) and LBL (Roberts and Goldstein, 1984), and the antibody CB (Furukawa et al., 1985) bind to all known blood group A variants, recognizing the terminal $\alpha$-GalNAc, and accommodating a wide range of subterminal saccharides. Antibodies Lu81, Cu 1, HH1, and HH5 also bind to $\alpha$-GalNAc, but recognize a narrower range of subterminal structures (Hirohashi et al., 1985). Antibody M1/22.25 recognizes the Forssman antigen, a blood group A-related structure in which a terminal GalNAc is $\alpha$-linked to a subterminal GalNAc instead of to galactose (Stern et al., 1978). None of these lectins or antibodies recognize the Cad antigen, but LBL, 

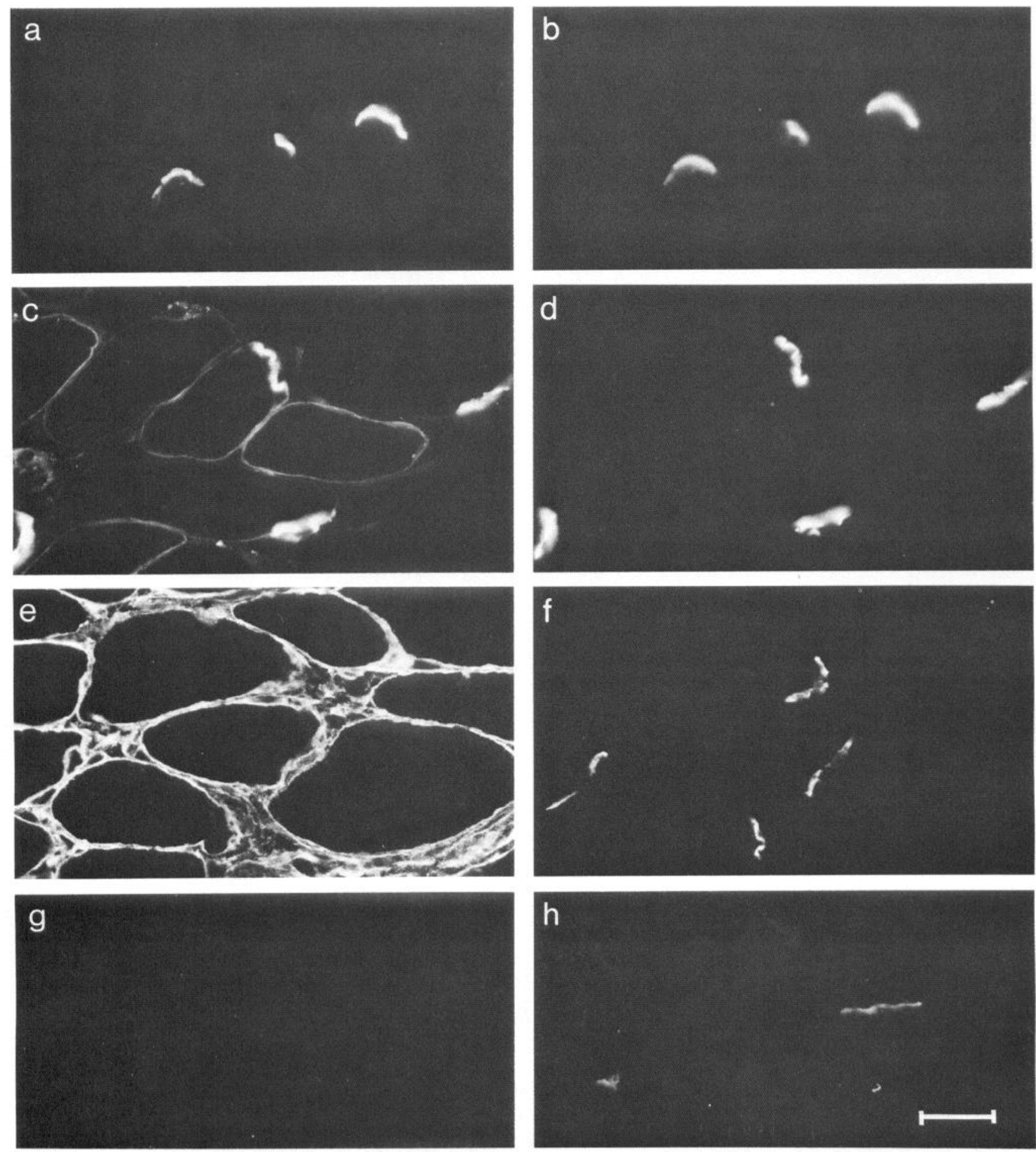

Figure 1. Cross sections of rat skeletal muscle doubly stained with biotinylated lectins or antibodies, followed by fluorescein-avidin or fluoresceinsecond antibody plus rhodamine- $\alpha$-bungarotoxin. Fluorescein optics (left panels) shows lectin or antibody staining, while illumination of the same fields with rhodamine optics (right panels) reveals $\alpha$-bungarotoxin-stained endplates. $a, b$, DBA stains synaptic but not extrasynaptic areas. $c, d$, HPA stains synapses intensely and extrasynaptic surfaces faintly. $e, f$, PHA-E stains synaptic and extrasynaptic areas to a similar extent. $g$, $h$, Antibody Lu81 stains neither synaptic nor extrasynaptic portions of the muscle fiber. Bar, $20 \mu \mathrm{m}$.

$\mathrm{CB}$, and Lu81 are known to recognize the Tn antigen, in addition to the blood group A antigen (Roberts and Goldstein, 1984; Furukawa et al., 1985; Hirohashi et al., 1985). None of these probes stained either synaptic or extrasynaptic portions of the muscle fiber surface (Fig. 1, $g, h$; Table 2), although all stained appropriate control tissues. These results argue that the synaptic carbohydrate is neither an $\alpha$-linked nor an O-linked anomer of GalNAc.

In contrast, $\mathrm{VVA}-\mathrm{B}_{4}$ and SSA, lectins that recognize $\beta$-GalNAc and O-linked GalNAc, but not $\alpha$-GalNAc (Bird and Wingham, 

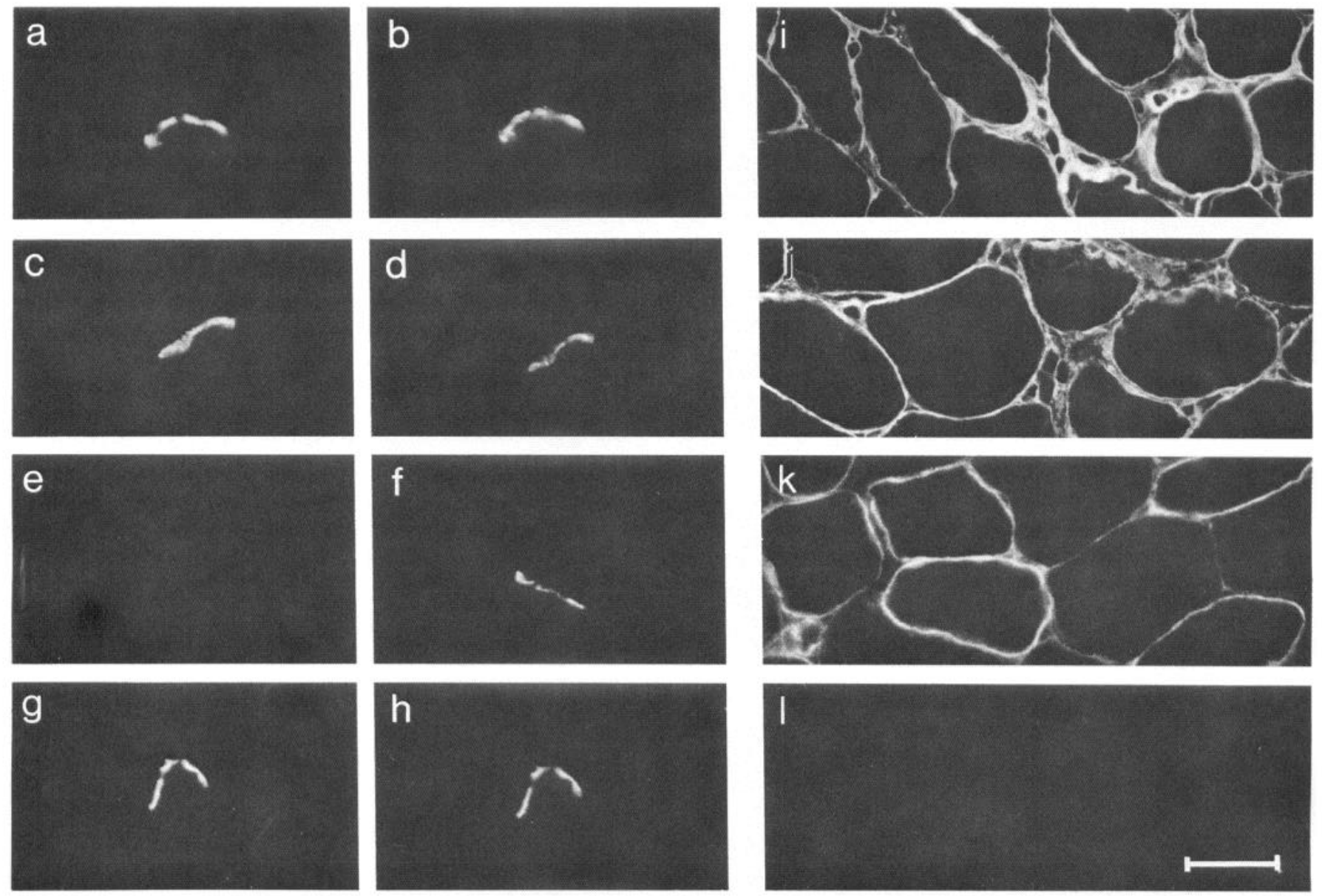

Figure 2. Effects of glycosidase digestion on lectin staining of muscle sections. Sections stained with biotin-lectin, fluorescein-avidin, and rhodamine- $\alpha$-bungarotoxin were viewed with fluorescein $(a, c, e, g, i-l)$ or rhodamine $(b, d, f, h)$ optics. Endplates of untreated muscle sections are selectively stained by VVA-B $\mathrm{B}_{4}(a, b)$. Digestion of sections with $\alpha-N$-acetylgalactosaminidase does not diminish endplate staining by VVA-B $(c$, $d$ ). In contrast, digestion of sections with jack bean $\beta-N$-acetylhexosaminidase abolishes VVA-B ${ }_{4}$ staining $(e, f)$, but does not affect staining by Con A $\left(i\right.$, control; $j$, digested). $\beta$-Galactosidase had no effect on VVA-B ${ }_{4}$ staining $(g, h)$, but did abolish staining by PNA $(k$, control; $l$, digested), a lectin that recognizes terminal $\beta$-galactose. Bar, $20 \mu \mathrm{m}$.

1974; Tollefsen and Kornfeld, 1983, 1984; Piller et al., 1986), stained synaptic sites selectively (Table 2). Staining by VVA$\mathrm{B}_{4}$, which agglutinates both $\mathrm{Tn}$ and $\mathrm{Cad} / \mathrm{Sda}$ erythrocytes strongly, was intense, while staining by SSA, which agglutinates Tn erythrocytes more strongly than $\mathrm{Cad} / \mathrm{Sda}$, was just as specific as VVA-B B $_{4}$ and DBA, but weaker. Together with the failure of $\alpha$-GalNAc and O-linked GalNAc-specific reagents to stain synapses, these results provide strong evidence that the synapsespecific carbohydrate is a terminal, $\beta$-linked GalNAc.

The availability of highly specific glycosidases provides a second, independent approach to the characterization of the GalNAc-containing synaptic carbohydrate. We digested muscle sections with $\alpha$-N-acetylgalactosaminidase, $\beta$ - $N$-acetylhexosaminidase, or $\beta$-galactosidase, then stained with DBA or VVA$\mathrm{B}_{4}$ to see which enzyme treatment affected staining. $\alpha$ - $N$-Acetylgalactosaminidase, which cleaves both $\alpha$ - and O-linked GalNAc (Hirohashi, 1985), had no effect on staining (Fig. 2). In contrast, $\beta$ - $N$-acetylhexosaminidase completely abolished

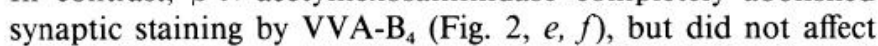
staining by Con A (Fig. 2, $i, j$ ), RCA, PHA-E, or PNA. $\beta$-Galactosidase (at concentrations 1000 -fold higher than were used for $\beta$ - $N$-acetylhexosaminidase) had no effect on synaptic

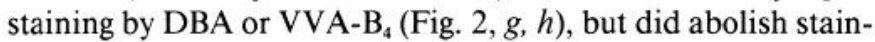

ing by PNA (Fig. 2, $k, l$ ), a lectin that recognizes terminal, $\beta$-linked galactose. These results confirm that the carbohydrate concentrated at the neuromuscular junction and recognized by

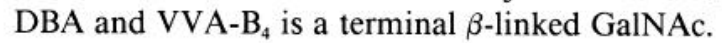

\section{Staining of neuromuscular junctions by $V V A-B_{4}$ in rat and other vertebrates}

Staining of synaptic sites by VVA-B $\mathrm{B}_{4}$ was qualitatively indistinguishable from, but somewhat more intense than, staining previously reported (Sanes and Cheney, 1982) for DBA. For this reason, and because $\mathrm{VVA}^{-B_{4}}$ proved to be more reliably active than DBA following derivatization to biotin or agarose, we characterized the staining of neuromuscular junctions by this lectin in further detail. Synaptic sites were intensely stained when whole muscles were incubated with biotin-VVA-B $\mathrm{B}_{4}$ and HRPavidin (Fig. 3, $a, b$ ), demonstrating that a major fraction of the glycoconjugate recognized by this lectin is external to the protein-impermeable plasma membrane. Electron microscopy of muscles stained in this way revealed reaction product concentrated in the synaptic cleft and junctional folds (Fig. $3 \mathrm{c}$ ); perisynaptic areas were occasionally lightly stained, while extrasynaptic surfaces, the Schwann cell surface, and the Schwann cellnerve terminal interface (which is accessible to immunochem- 

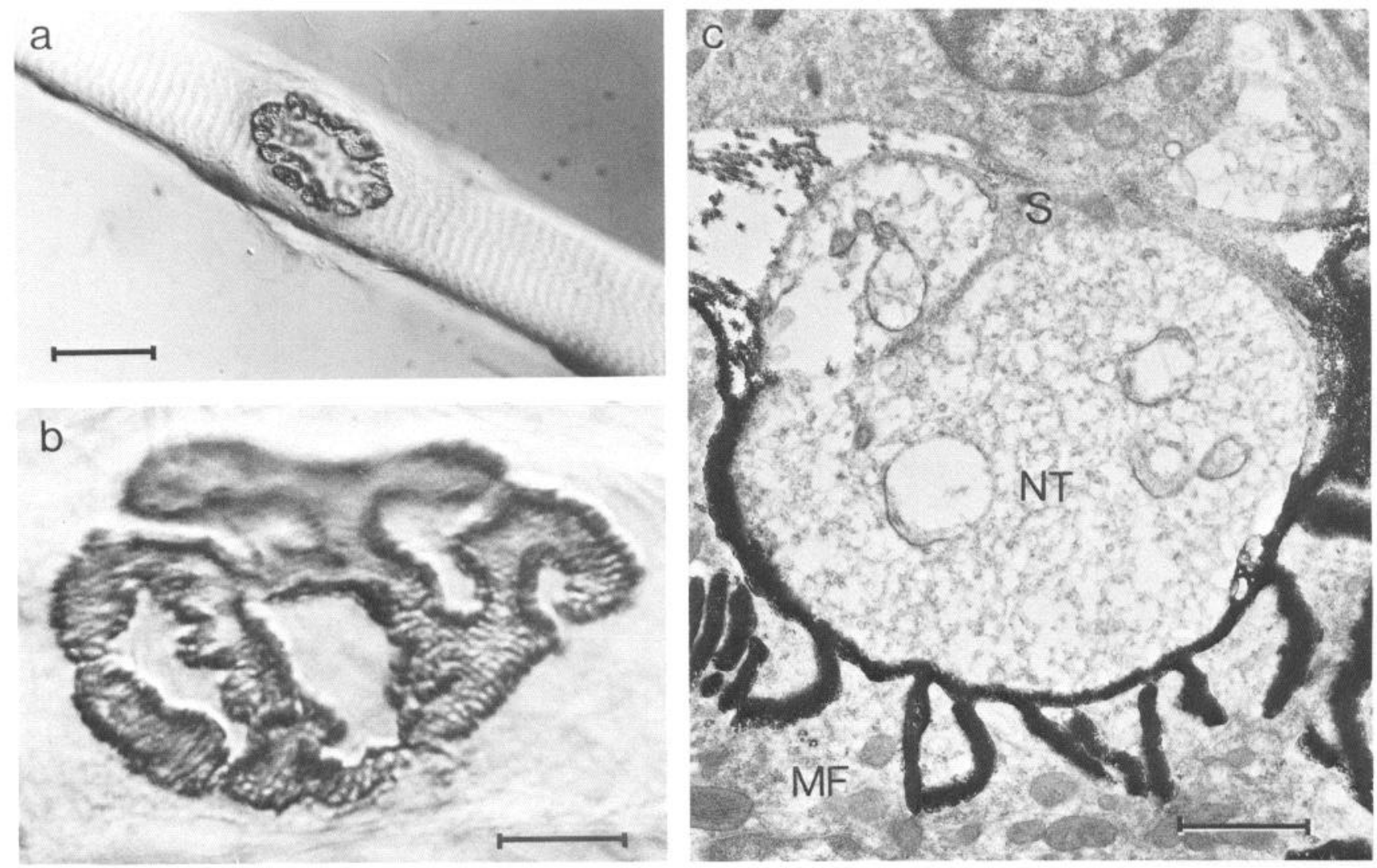

Figure 3. VVA-B S $_{4}$ selectively stains the neuromuscular junction. A rat muscle was incubated with biotin-VVA-B $\mathrm{B}_{4}$ and HRP-avidin, then fixed and stained for HRP. Endplates are selectively stained on single fibers teased from the muscle $(a)$, and outlines of junctional folds are visible at higher magnification $(b)$. Electron microscopy reveals reaction product concentrated in the synaptic cleft and junctional folds; perisynaptic areas are sometimes lightly stained, while extrasynaptic and Schwann cell surfaces are unstained $(c)$. $S$, Schwann cell; $N T$, nerve terminal; $M F$, muscle fiber. Bars: $50 \mu \mathrm{m}(a) ; 10 \mu \mathrm{m}(b) ; 1 \mu \mathrm{m}(c)$.

ical reagents; see Covault and Sanes, 1986) were unstained. Thus, VVA- $\mathrm{B}_{4}$ binds to a glycoconjugate that is concentrated in the area of apposition between nerve and muscle at the neuromuscular junction. A major fraction of this glycoconjugate is associated with the muscle fiber surface, since the intensity of staining is not detectably decreased in denervated muscle (not shown), but we cannot exclude the possibility that some VVA$\mathrm{B}_{4}$-binding molecules are also associated with the nerve terminal membrane.

To examine whether the VVA- $\mathrm{B}_{4}$-binding glycoconjugate is a general marker of neuromuscular junctions, we stained cryostat sections of skeletal muscles from a variety of vertebrate species. As summarized in Table $3, \mathrm{VVA}-\mathrm{B}_{4}$ selectively stained synaptic sites in muscles of all major vertebrate classes except chondrichthyes, although in several species extrasynaptic portions of the muscle fiber surface were faintly stained as well (e.g., Fig. 4). In rat, we stained a series of muscles that varied in position and fiber-type composition. The specificity of synaptic staining was similar in soleus (a predominantly slow-twitch limb muscle), extensor digitorum longus (a predominantly fast-twitch limb muscle), internal intercostal, external intercostal, platysma, and diaphragm muscles (axial muscles of varying fiber-type proportions). Thus, VVA-B $\mathrm{B}_{4}$ marks synaptic sites in a variety of vertebrate skeletal muscles.

\section{Lectin receptors}

Three types of glycoconjugates are known to bear GalNAc residues linked in the $\beta$-anomeric form-glycoproteins, glycolipids, and glycosaminoglycans. We tested synapse-associated molecules in each class for their ability to bind VVA-B $B_{4}$ and DBA.

\section{AChE}

$\mathrm{AChE}$ is an easily assayed glycoprotein that, like VVA- $\mathrm{B}_{4}$ and DBA-binding material, is highly concentrated in the basal lamina of the neuromuscular junction. To determine whether VVA$\mathrm{B}_{4}$ or DBA bound $\mathrm{AChE}$, extracts from rat diaphragm were incubated with lectin-agarose, and the fraction of the AChE that bound was determined. A significant fraction of the AChE activity in the extract bound to DBA- (Fig. 5) or VVA-B - $_{4}$-agarose. This binding was inhibited by GalNAc but not by another saccharide, $\alpha$-methylmannoside. No AChE activity bound to unconjugated agarose. Thus, DBA and VVA- $\mathrm{B}_{4}$ recognize $\mathrm{AChE}$ from muscle.

In these experiments, less than $20-25 \%$ of the $\mathrm{AChE}$ in the extract bound to DBA or VVA- $\mathrm{B}_{4}$ even when lectin-agarose was added in excess. By contrast, $60-90 \%$ of the AChE bound to Con A-agarose and was therefore glycosylated (Fig. 5). This observation raised the possibility that muscle $\mathrm{AChE}$ is heterogeneous in its pattern of glycosylation (see Rotundo, 1984). $\mathrm{AChE}$ is known to exist in several different forms in muscle (reviewed in Massoulie and Bon, 1982). These forms can be divided into 2 groups, globular and asymmetric. In rat muscle, globular AChE exists as monomers $\left(G_{1}\right)$, dimers $\left(G_{2}\right)$, or tetramers $\left(G_{4}\right)$ of the catalytic subunits, and is present at low levels throughout the muscle fiber. Asymmetric AChE consists of one $\left(A_{4}\right), 2\left(A_{8}\right)$, or $3\left(A_{12}\right)$ of the $G_{4}$ tetramers covalently linked to 

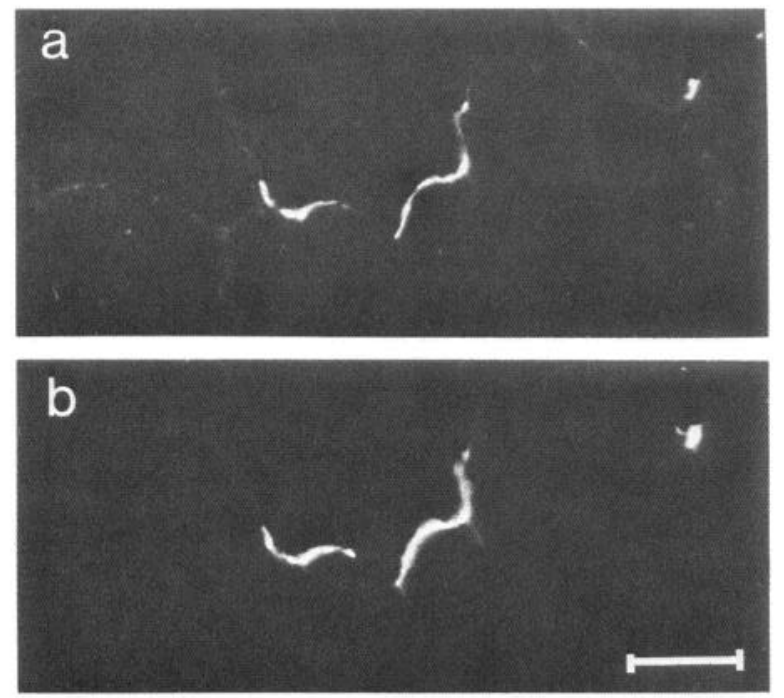

Figure 4. Selective staining of goldfish neuromuscular junctions by VVA- $B_{4}$. Section was stained with biotin-VVA- $B_{4}$, fluorescein-avidin $(a)$, and rhodamine- $\alpha$-bungarotoxin $(b)$. Bar, $20 \mu \mathrm{m}$.

a collagenlike tail. The asymmetric forms, predominantly $\mathrm{A}_{12}$, are concentrated at or near the neuromuscular junction (Hall, 1973; Younkin et al., 1982), and may be anchored in the basal lamina of the synaptic cleft (McMahan et al., 1978).

To see whether the asymmetric (A) and globular (G) forms of AChE bound differently to VVA-B $\mathrm{B}_{4}$ and DBA, we used sucrose-gradient centrifugation to analyze $\mathrm{AChE}$ applied to and

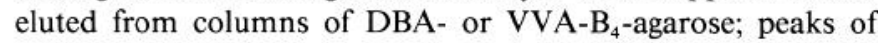
activity sedimenting at $4,10,12$, and $16 \mathrm{~S}$ are known to correspond to the $G_{1}, G_{4}, A_{8}$, and $A_{12}$ forms, respectively (Massoulie and Bon, 1982; Fig. 6a). Results from a DBA-agarose column are shown in Figure $6 b$. No AChE activity was eluted with $50 \mathrm{~mm} \alpha$-methylmannoside, but a peak of activity was eluted with $50 \mathrm{~mm}$ GalNAc. When analyzed on a $5-20 \%$ isokinetic sucrose gradient, this peak was found to be composed exclusively of asymmetric $\mathrm{AChE}\left(\mathrm{A}_{8}\right.$ and $\mathrm{A}_{12}$; Fig. $6 c$ ). To insure that asymmetric AChE was selectively bound to, rather than preferentially eluted from, the column, we isolated muscle extracts enriched in either G-forms or A-forms. Low-salt buffers with detergent selectively solubilize the G-forms in muscle, and subsequent extraction of the residual pellet with buffer containing $1 \mathrm{M} \mathrm{NaCl}$ solubilizes the A-forms (Younkin et al., 1982; Fig. $7, A, B)$. Up to $70 \%$ of the AChE activity in the A-rich extracts

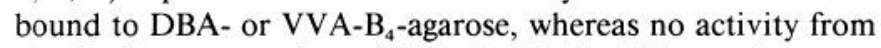
the G-rich extracts bound (Fig. 7, $C, D$ ). This confirms our conclusion that the asymmetric, synapse-specific forms of $\mathrm{AChE}$, but not the globular forms, are recognized by DBA and VVA$\mathrm{B}_{4}$.

In 2 experiments, we considered the extent to which binding of AChE to VVA-B ${ }_{4}$ and DBA is selective. First, the specific activities of $\mathrm{AChE}$ (U/mg protein) in a muscle extract and in the eluate from a lectin-agarose column were determined. A purification of $>200$-fold resulted from the chromatographic step, indicating that most proteins in muscle did not bind to the gel. Second, a ${ }^{125} \mathrm{I}$-glycoprotein fraction from muscle, containing $\mathrm{AChE}$, was iodinated and subjected to chromatography on VVA-B ${ }_{4}$-agarose. Approximately $20 \%$ of the AChE applied, but $\leq 0.1 \%$ of the ${ }^{125}$ I-labeled material bound to the column and was eluted by GalNAc (Fig. 8). Thus, as expected from the

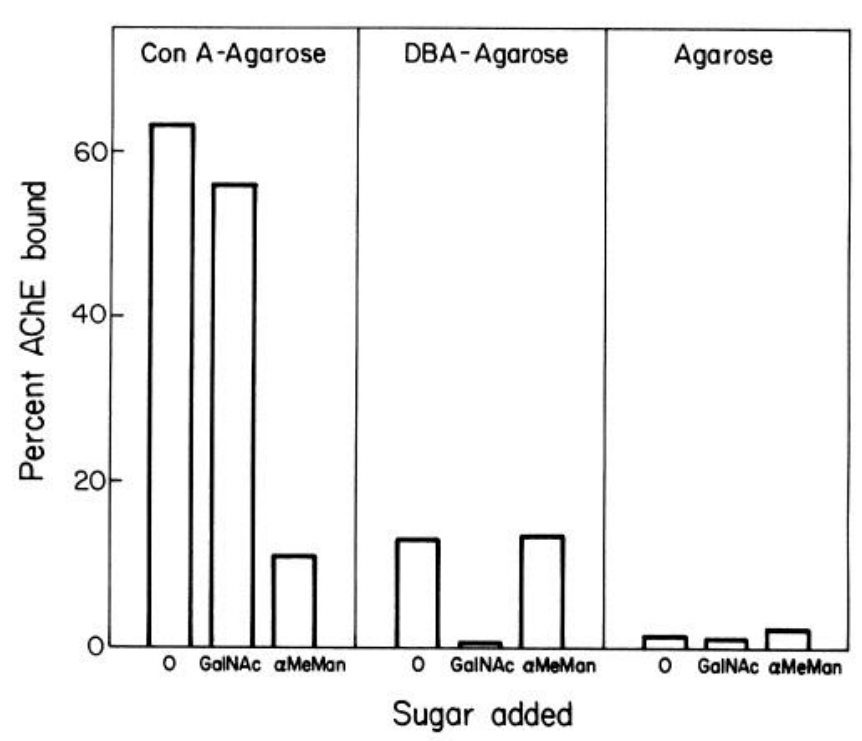

Figure 5. Binding of muscle AChE to lectin-agarose. Aliquots of a muscle extract were mixed with agarose conjugates and sugars ( $200 \mathrm{~mm}$ ) as indicated, and incubated for $12 \mathrm{hr}$ at $4^{\circ} \mathrm{C}$. Percentage binding was calculated as described in Materials and Methods.

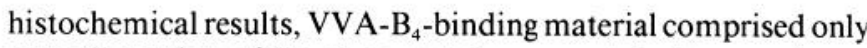
a small portion of the glycoproteins present in the muscle extract.

A synaptic glycolipid. Globoseries glycolipids and gangliosides that terminate in $\beta$-GalNAc are known to be membrane components in a variety of tissues, including muscle (Svennerholm et al., 1972; Chien and Hogan, 1983). We therefore stained sections of rat muscle with 3 monoclonal antibodies to GalNAccontaining lipids: anti-stage-specific embryonic antigens (SSEA)-3 and -4 (Shevinsky et al., 1982; Kannagi et al., 1983a), and anti-GM2 (Natoli et al., 1986). GM2 has the structure GalNAc $\beta 1$ - 4[NeuAc $\alpha 2-3]$ Gal $\beta 1$ - 1Ceramide; anti-SSEA-3 binds to globoseries glycolipids with the common structure $\mathrm{R}-3 \mathrm{GalNAc} \beta 1-3 \mathrm{Gal} \alpha 1-4 \mathrm{R}^{\prime}$, as well as to some glycoproteins; and anti-SSEA-4 binds to glycolipids in which the SSEA-3 antigen is extended by a galactose and a sialic acid residue $(\mathrm{R}=$ NeuAc $\alpha 2-3 \mathrm{Gal} \beta 1-3)$.

Anti-SSEA-3 selectively stained synaptic sites in rat skeletal muscle (Fig. 9, $a, b$ ); nonsynaptic portions of the muscle fiber, and intramuscular structures such as blood vessels and nerve trunks, were very faintly stained. Anti-GM2 also stained synaptic but not extrasynaptic portions of the muscle fiber surface (not shown). Synaptic sites were occasionally stained by antiSSEA-4, but staining was too faint to be characterized. Staining by anti-SSEA-3 and anti-GM2 (but not by VVA-B H $_{4}$ was abolished by pretreatment of sections with chloroform-methanol (Fig. 9, $c, d$ ), which selectively extracts lipids and is commonly used as a diagnostic test for their presence (see, for example, Dodd et al., 1984). Thus, glycolipid-like molecules bearing $\beta$-GalNAc residues are concentrated at the neuromuscular junction.

To ask whether the synaptic antigens recognized by antiSSEA-3 or anti-GM2 are VVA- B $_{4}$-binding glycoconjugates, we preincubated muscle sections with $1 \mathrm{mg} / \mathrm{ml}$ unconjugated VVA$\mathrm{B}_{4}$, then stained the sections with antibody. VVA-B $\mathrm{B}_{4}$ blocked endplate staining with anti-SSEA-3 (Fig. 9, $e, f$ ) and anti-GM2, demonstrating that the lectin recognizes the same synaptic molecules as do anti-SSEA-3 and anti-GM2, or closely associated 

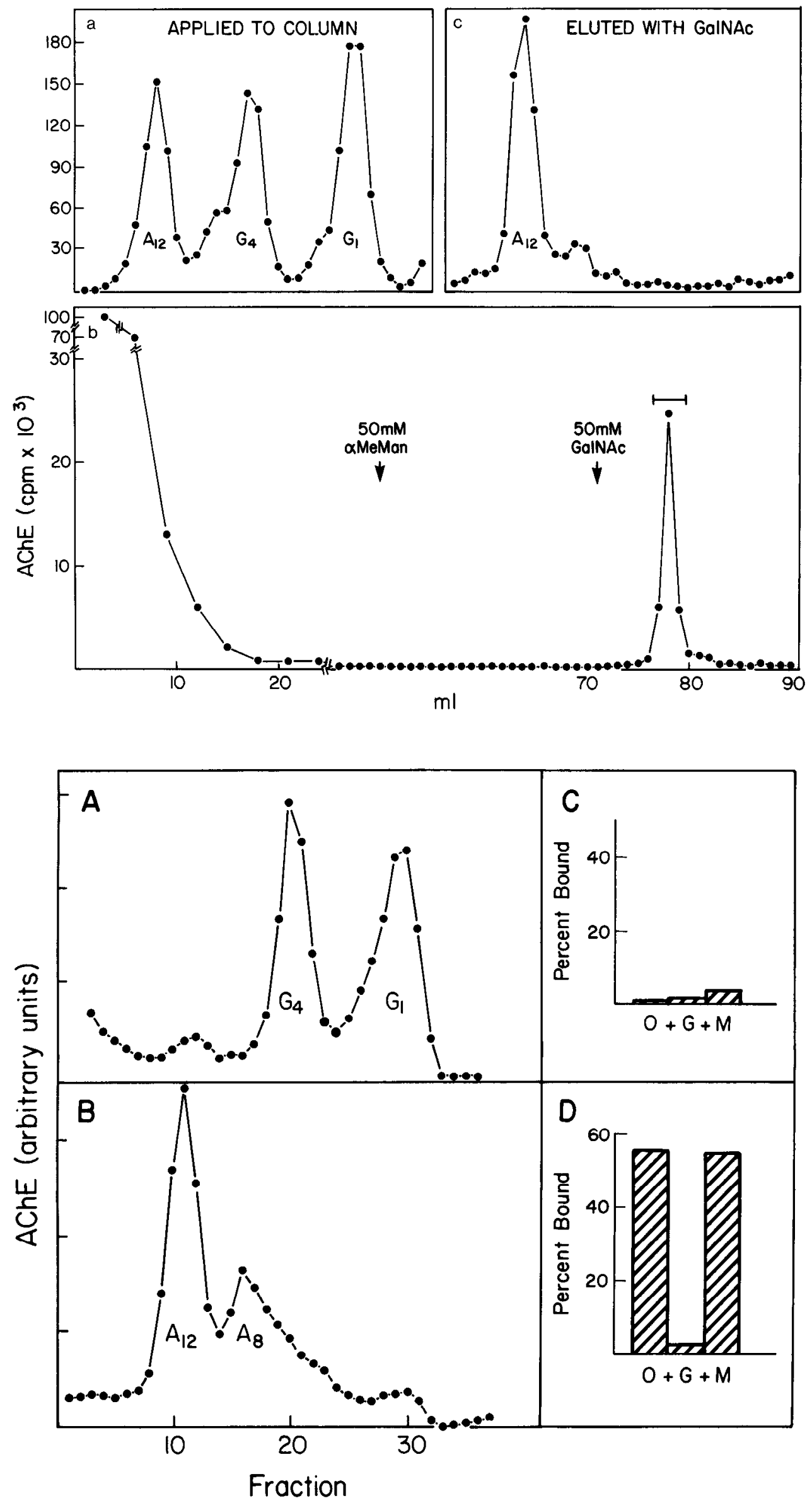

Figure 6. Chromatography of muscle AChE on a column of DBA-agarose. $a$, Sedimentation analysis of the "highsalt" extract that was applied to the column. The gradient profile shows that multiple forms of AChE are present. The major forms were identified by their sedimentation coefficients (see Massoulie and Bon, 1982). $b$, Elution profile shows that some AChE was specifically eluted from the column with GalNAc. $c$, Sedimentation analysis of the eluted peak (bar in $b$ ) demonstrates that the AChE eluted by GalNAc was the $A_{12}$ form. Similar results have been obtained with VVA-B . $_{4}$
Figure 7. Binding of asymmetric and globular AChE to VVA-B 4 . G-rich and A-rich extracts were prepared as described in Materials and Methods. Sedimentation analysis indicates the enrichment for globular $(A)$ and asymmetric $(B)$ forms achieved by the selective extraction protocol used. Incubation of the extracts with $V \vee A-B_{4}-$ agarose, as described in the Figure 4 legend, showed that globular forms $(C)$ do not bind, but that asymmetric forms $(D)$ do bind to VVA-B . $_{4}$ 


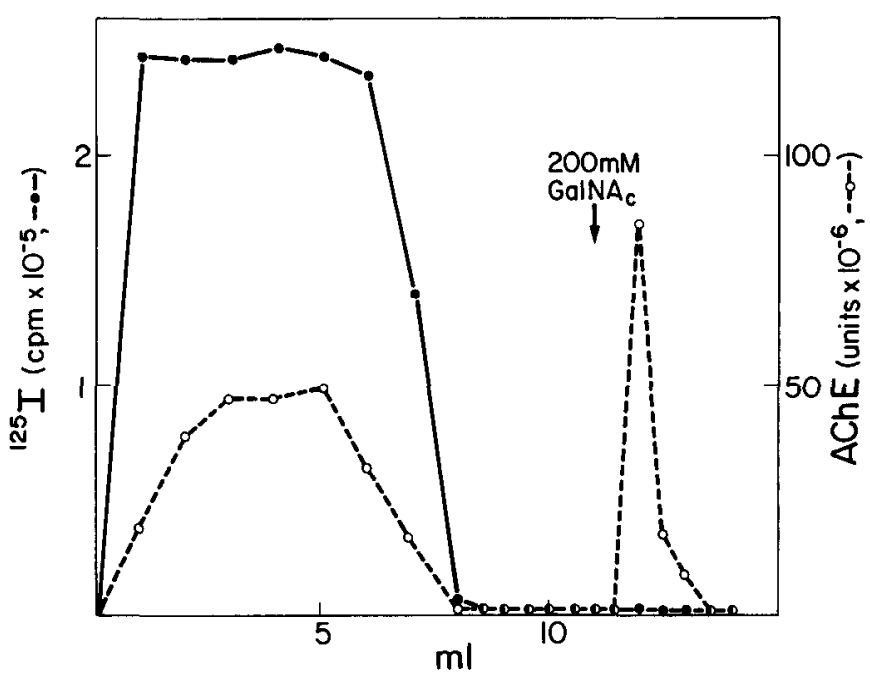

Figure 8. Separation of AChE from other glycoproteins by chroma-

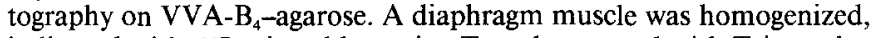
iodinated with ${ }^{125} \mathrm{I}$ using chloromine $\mathrm{T}$, and extracted with Triton plus high-salt buffer (see Materials and Methods). The extract was fractionated on Con A-agarose to isolate glycoproteins. Material eluted with $500 \mathrm{~mm} \alpha$-methylmannoside, the glycoprotein fraction, was applied to a VVA-B ${ }_{4}$-agarose column. Little ${ }^{125}$ I but $\sim 20 \%$ of the AChE applied was retained by the column and eluted by GalNAc.

molecules. Do these antibodies recognize AChE? Synaptic sites were intensely stained by anti-AChE antiserum (and by all of a large number of antibodies to known glycoprotein components of the muscle membrane and basal lamina) following chloroform-methanol treatment of sections, while staining by antiSSEA-3 and anti-GM2 was abolished. Furthermore, removal of the muscle fiber plasma membrane by damage-induced degeneration of muscle (Sanes and Hall, 1979) abolished antiSSEA-3 and anti-GM2-like immunoreactivity without greatly affecting the intensity of staining by anti-AChE or other anti- bodies to BL. VVA-B ${ }_{4}$ staining survived both of these treatments. Thus, $\mathrm{AChE}$ and glycolipid-like molecules are distinct receptors for $\mathrm{VVA} \mathrm{B}_{\uparrow}$ at the neuromuscular junction.

\section{Glycosaminoglycans}

The glycosaminoglycan chondroitin is composed of the repeating disaccharide (GalNAc $\beta 1-4$ Glucuronate $\beta 1-3$ ). Because skeletal muscle is known to synthesize chondroitin and its sulfated derivatives (Ahrens et al., 1977; Carrino and Caplan, 1982), we asked whether these structures are recognized by DBA or VVA$B_{4}$. First, we mixed chondroitin, chondroitin-4-sulfate, chondroitin-6-sulfate, or dermatan sulfate (chondroitin sulfate B) with biotinylated lectin to see whether this would block its staining of neuromuscular junctions. In addition, we tested heparin and heparan sulfate in light of a report that a GalNAc-inhibitable lectin isolated from chick embryos binds heparin (Ceri et al., 1981). However, none of these glycosaminoglycans, at concentrations of up to $1 \mathrm{mg} / \mathrm{ml}$, detectably affected staining by DBA or VVA-B ${ }_{4}$, whereas $10 \mu \mathrm{g} / \mathrm{ml}$ of porcine gastric mucin, which is rich in terminal GalNAc (Hounsell and Feizi, 1982) completely blocked staining. Second, we stained muscles with a series of 3 monoclonal antibodies ( $1 \mathrm{~B} 5,9 \mathrm{~A} 2$, and $3 \mathrm{~B} 3$ ) that recognize chondroitin, chondroitin-4-sulfate, and chondroitin6-sulfate, respectively (Couchman et al., 1984). Dermatan sulfate is also recognized by $9 \mathrm{~A} 2$; this sulfate is the only GalNAcrich glycosaminoglycan other than the chondroitins. None of these antibodies stains the neuromuscular junction selectively: anti-chondroitin-6-sulfate (Fig. 10) and anti-chondroitin stained both synaptic and extrasynaptic areas, while anti-chondroitin4-sulfate stained neither synaptic nor extrasynaptic areas. Thus, VVA- $B_{4}$ and DBA appear not to recognize the $\beta$-GalNAc in chondroitin, and chondroitin appears not to be concentrated at the neuromuscular junction.

\section{Discussion}

The histochemical evidence that the synapse-specific carbohydrate initially defined by DBA (Sanes and Cheney, 1982) is a

Table 3. Staining of synaptic and extrasynaptic portions of skeletal muscle fiber surfaces by VVA-B in various vertebrate species

\begin{tabular}{|c|c|c|c|c|}
\hline Class & Species & Muscle & $\begin{array}{l}\text { Synaptic } \\
\text { sites }\end{array}$ & $\begin{array}{l}\text { Extra- } \\
\text { synaptic } \\
\text { areas } \\
\end{array}$ \\
\hline \multirow[t]{10}{*}{ Mammalia } & \multirow[t]{6}{*}{ Rat } & Diaphragm & +++ & - \\
\hline & & Internal intercostal & +++ & - \\
\hline & & External intercostal & +++ & - \\
\hline & & Platysma & +++ & - \\
\hline & & Solcus & +++ & - \\
\hline & & Extensor digitorum longus & +++ & - \\
\hline & Guinea pig & Diaphragm & +++ & - \\
\hline & Mouse & Diaphragm & +++ & - \\
\hline & Rabbit & Diaphragm & +++ & \pm \\
\hline & Human & Intercostal & +++ & + \\
\hline Aves & Chick & Pectoralis & +++ & \pm \\
\hline Reptilia & Garter snake & Intercostal & +++ & \pm \\
\hline \multirow[t]{2}{*}{ Amphibia } & Frog (Rana pipiens) & Sartorius & $t+t$ & \pm \\
\hline & Axolotl & Iliotibialis & +++ & + \\
\hline Osteichthyes & Goldfish & Axial & +++ & \pm \\
\hline Chondrichthyes & Torpedo californica & Tail & +++ & +++ \\
\hline Cyclostomata & Lamprey & Ventral body wall & +++ & - \\
\hline
\end{tabular}



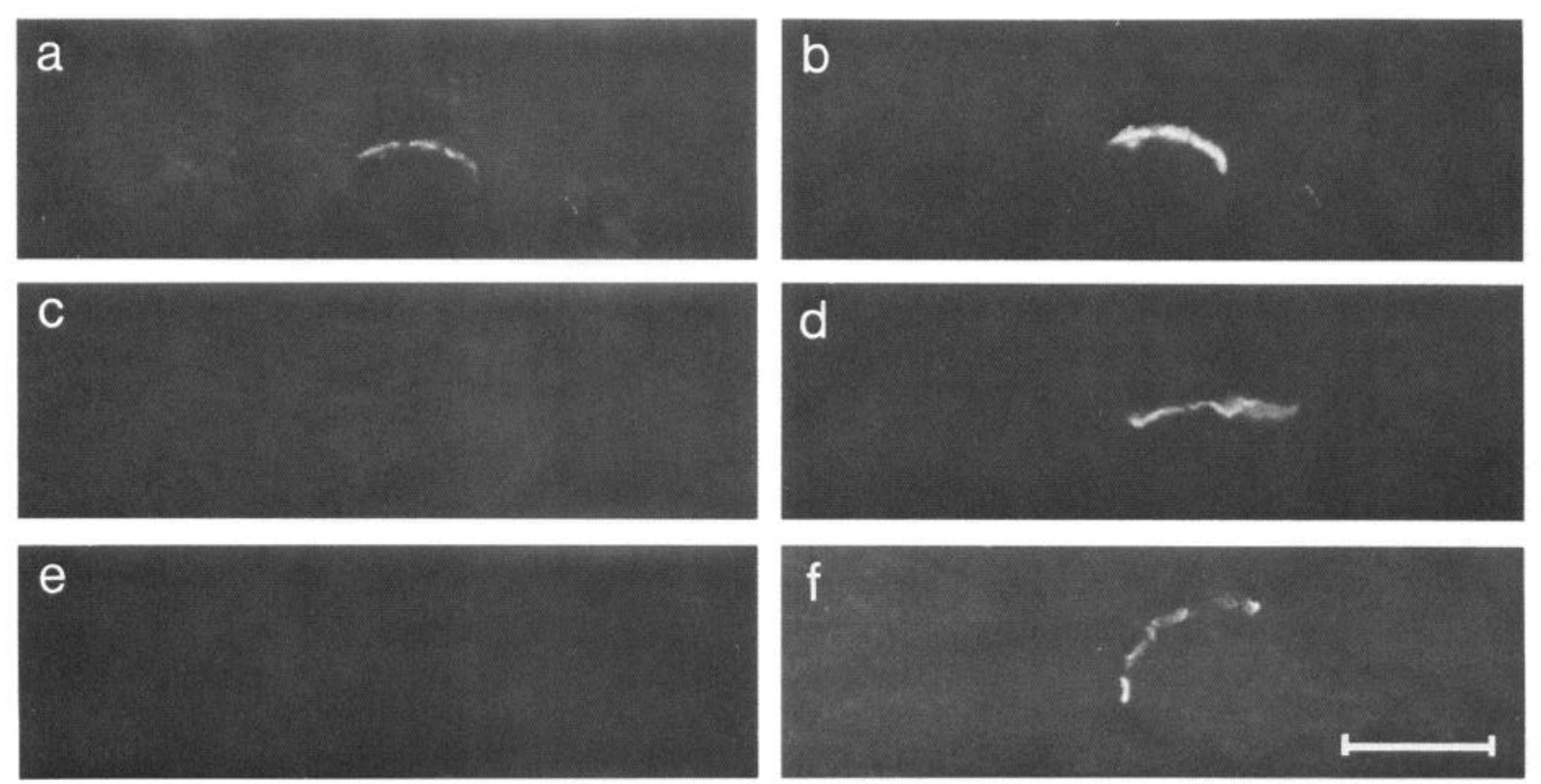

Figure 9. Cross sections of rat skeletal muscle, doubly stained with anti-SSEA-3 and fluorescein-second antibody ( $a, c, e)$ plus rhodamine- $\alpha$ bungarotoxin $(b, d, f), c, d$, Section was incubated with VVA-B ${ }_{4}$ before and during staining, while the section in $e, f$ was incubated with chloroform/ methanol before staining. Anti-SSEA-3 specifically stains synaptic sites; staining is inhibited by VVA-B ${ }_{4}$ and abolished by pretreatment with chloroform/methanol. Bar, $20 \mu \mathrm{m}$.

$\beta$-GalNAc-terminated saccharide can be summarized as follows: (1) Lectins specific for GalNAc stain synaptic sites specifically, lectins that prefer GalNAc to galactose stain synaptic sites more intensely than they do extrasynaptic areas, and lectins that do not recognize GalNAc do not distinguish between synaptic and extrasynaptic areas. (2) Lectins and antibodies that recognize $\alpha$-GalNAc and/or O-linked GalNAc, but not $\beta$-GalNAc, do not stain synaptic sites. (3) Lectins that recognize $\beta$-GalNAc and O-linked GalNAc but not $\alpha$-GalNAc stain synaptic sites selectively. (4) A lectin that prefers O-linked GalNAc to $\beta$-GalNAc, but that recognizes both, stains synaptic sites selectively but weakly. (5) Staining of synapses by VVA-B $B_{4}$ or DBA is abolished by pretreatment of sections with $\beta-N$-acetylhexosaminidase but not by $\alpha-N$-acetylgalactosaminidase (which cleaves both $\alpha$-GalNAc and O-linked GalNAc) or $\beta$-galactosidase. This evidence is limited by the incompletely defined specificities of the probes we used (see, for example, Debray et al., 1981), and we cannot exclude the possibility that other sugars or GalNAc in other linkages (e.g., the phosphodiester linkage; Balsamo et al., 1986) contribute to the synapse-specific carbo-
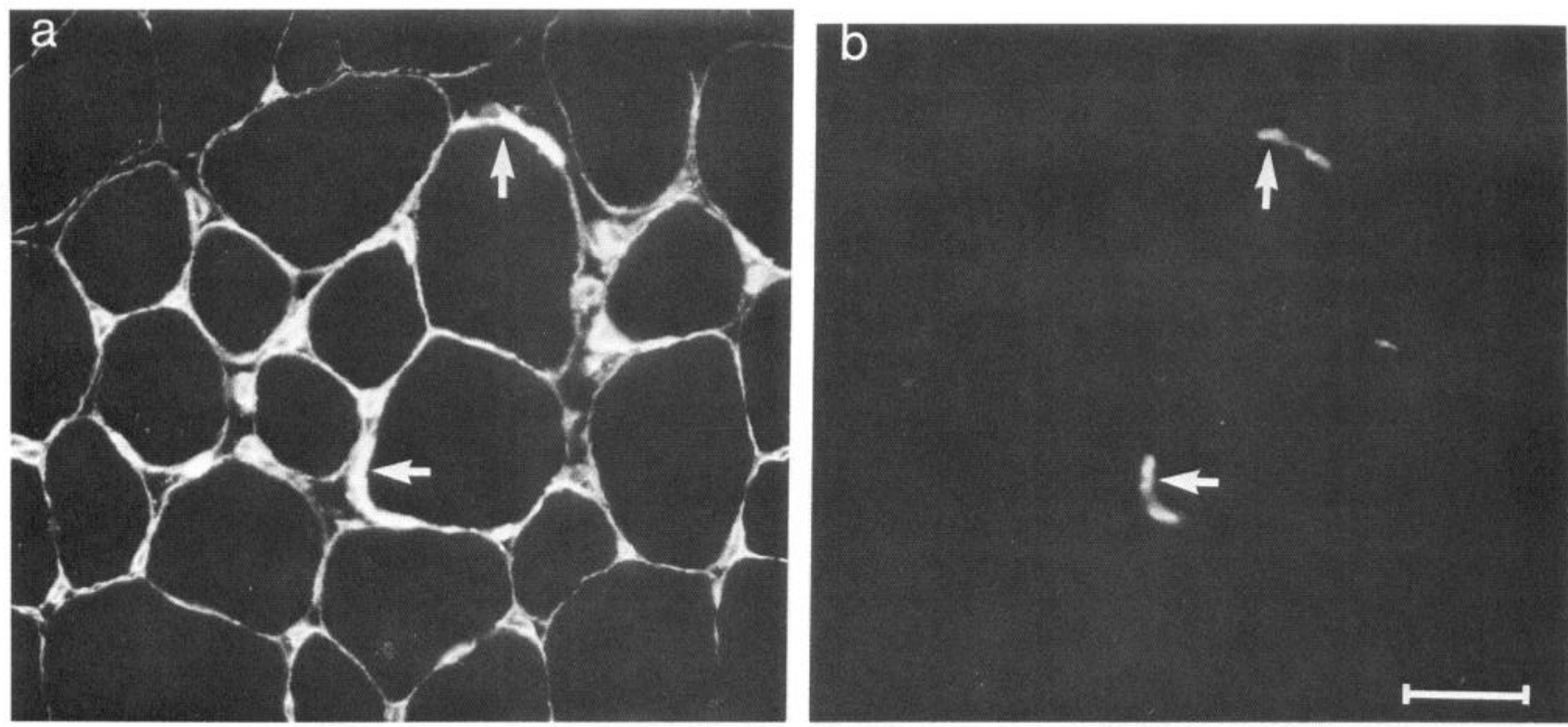

Figure 10. Cryostat section of rat skeletal muscle doubly stained with monoclonal antibody to chondroitin-6-sulfate (3B3) and fluorescein-second antibody $(a)$ plus rhodamine $\alpha$-bungarotoxin $(b)$. Both synaptic and extrasynaptic portions of the muscle fiber surface are rich in chondroitin-6sulfate-like material. Bar, $20 \mu \mathrm{m}$. 
hydrate. Nonetheless, the consistent results obtained with a variety of reagents give us some measure of confidence in our conclusion.

Using VVA-B ${ }_{4}$, we have shown that terminal GalNAc residues are concentrated at neuromuscular junctions in several mammals and in representatives of 6 of the 7 major vertebrate classes (see Storer et al., 1972). In some species, including humans, staining of extrasynaptic as well as synaptic areas is detectable, but in each case binding sites are considerably enriched at the synapse. One exception to this generalization is Torpedo, in which both synaptic and extrasynaptic areas are stained by VVA- $B_{4}$. While we have no explanation for this difference between Torpedo and other vertebrates, it is possible that $\beta$-GalNAc is concentrated at synapses but that another VVA-B $\mathrm{B}_{4}$-binding glycoconjugate (e.g., one containing O-linked GalNAc) is present throughout the muscle fiber surface. In any event, the conservation of GalNAc as a selective marker of synaptic sites in the muscles of all but one of the 12 species tested is in marked contrast with the variability of glycosylation patterns typically described in such comparative studies (see, for example, Holthoffer, 1983; Kobata, 1984; Mohan and Spiro, 1986). This conservation argues that the association of $\beta$-GalNAc with the synapse is of some functional significance.

Using lectin-agarose conjugates, we have demonstrated that asymmetric, collagen-tailed AChE binds to DBA and VVA-B, while globular $\mathrm{AChE}$ does not. AChE is highly concentrated at synaptic sites in rat muscle, but some enzyme is present extrasynaptically. The synapse-associated AChE is predominantly asymmetric, while the extrasynaptic enzyme is predominantly globular (Hall, 1973; Younkin et al., 1982). Thus, DBA and VVA- $_{4}$ recognize a synapse-specific form of a synapse-associated molecule. An obvious possibility is that these lectins bind to the collagenous tail that constitutes the distinguishing characteristic of asymmetric $\mathrm{AChE}$. It is also possible, however, that the lectins recognize the globular, catalytic subunit or a hitherto unidentified AChE-associated moiety; evidence for heterogenity of catalytic subunits (Doctor et al., 1983) and for the existence of additional subunits (Lee et al., 1982) has been reported for Torpedo AChE. Furthermore, in all of our experiments, a fraction of the asymmetric enzyme remained unbound to lectin. Whether incomplete binding is simply a consequence of the conditions of our assay, or whether it represents heterogeneity within the asymmetric pool (Barat et al., 1984) remains to be determined.

By using 2 antibodies, anti-SSEA- 3 and anti-GM2, that recognize $\beta$-GalNAc-terminated glycolipids, we defined a novel antigen concentrated at the neuromuscular junction. The abolition of staining by chloroform-methanol, the fine distribution of the antigen, and the known specificity of these antibodies for glycolipids all suggest that the neuromuscular antigens recognized by these antibodies are glycolipids. Abolition of SSEA-3and GM2-like immunoreactivity by treatments that spare $\mathrm{AChE}$ and VVA- $\mathrm{B}_{4}$-binding sites, and inhibition of staining by VVA$\mathrm{B}_{4}$ demonstrate that the glycolipid-like antigens bear the synapse-specific carbohydrate but are distinct from AChE. We do not know, however, whether anti-SSEA-3 and anti-GM2 bind to the same or to different glycolipids: it is unclear whether antiSSEA-3 recognizes GM2 or whether anti-GM2 might recognize some variants of SSEA-3 (Kannagi et al., 1983a, b; Natoli et al., 1986). Experiments to characterize the glycolipid(s) at the neuromuscular junction are now in progress. While no glycolipid has hitherto been shown to be concentrated at the neuromus- cular junction, it is interesting that Obata et al. (1977) showed that the glycolipid globoside, which is recognized by anti-SSEA-3 (Kannagi et al., 1983a; Tippett et al., 1986), can perturb normal synaptogenesis when added to nerve-muscle cocultures. Furthermore, anti-SSEA-3 has been shown to stain a restricted subset of sensory neurons in dorsal root ganglia, consistent with the suggestion that globoseries glyclipids may serve as "differentiation antigens" both within and outside of the nervous system (Dodd et al., 1984).

The result that 2 molecules share a synapse-specific localization and a synapse-specific carbohydrate invites speculation that these attributes are causally related. It is unlikely that the glycosylation results from synaptogenesis, because VVA- $\mathrm{B}_{4}$ stains extrasynaptic areas in embryonic and denervated muscles (L. C. Scoll and J. R. Sanes, unpublished observations). Alternatively, the synapse-specific carbohydrate might result in preferential localization at synaptic sites via interaction of $\beta$-GalNAc-bearing molecules with intracellular or extracellular $\beta$-GalNAc-binding molecules (endogenous lectins; see Lis and Sharon, 1986). One precedent for this is the targeting of acid hydrolases to the lysosome. Several lysosomal enzymes acquire the same unusual saccharide, a terminal mannose-6-phosphate, as they are synthesized. This carbohydrate serves as a common marker: an endogenous mannose-6-phosphate receptor binds these hydrolases and directs their transport to lysosomes (Sahagian, 1987). Thus, it is clear that carbohydrate structures can be involved in directing the movements of glycoconjugates, and it is reasonable to imagine that other targeting systems, with their own carbohydrate markers, exist (see Lis and Sharon, 1986). If this hypothesis were true, other synapse-specific molecules would bear the synapse-specific carbohydrate shared by $\mathrm{AChE}$ and the synaptic glycolipids.

\section{References}

Ahrens, P. B., M. Solursh, and S. Meier (1977) The synthesis and localization of glycosaminoglycans in striated muscle differentiating in cell culture. J. Exp. Zool. 202: 375-388.

Appukuttan, P. S., and D. Basu (1981) Isolation of $N$-acetylgalactosamine-binding protein from winged bean (Psophocarpus tetragonolobus). Anal. Biochem. 113: 253-255.

Askanas, V., H. Kwan, R. Alvarez, T. Kobayashi, A. Martinuzzi, and W. K. Engel (1986) De novo neuromuscular junction (NMJ) formation in human muscle cultured in monolayer and innervated by fetal rat spinal cord. Muscle Nerve (Suppl.) 9: 13.

Baker, D. A., S. Sugii, E. A. Kabat, R. M. Ratcliffe, P. Hermentin, and R. V. Lemieux (1983) Immunochemical studies on the combining sites of Forssman hapten reactive hemagglutinins from Dolichos biflorus, Helix pomatia, and Wisteria floribunda. Biochemistry 22: 27412750.

Balsamo, J., R. S. Pratt, and J. Lilien (1986) Chick neural retina $N$-acetylgalactosaminyltransferase/acceptor complex: Catalysis involves transfer of $\mathrm{N}$-acetylgalactosamine phosphate to endogenous acceptors. Biochemistry 25: 5402-5407.

Barat, A., J. Gomez-Barriocanal, and G. Ramirez (1984) Two classes of collagen-tailed, asymmetric molecular forms of acetylcholinesterase in skeletal muscle: Differential effects of denervation. Neurochem. Int. 6: 403-412.

Bird, G. W. G., and J. Wingham (1974) Haemagglutinins from Salvia. Vox Sang. 26: 163-166.

Blanchard, D., J.-P. Cartron, B. Fournet, J. Montreuil, H. van Halbeek, and J. F. E. Vliegenthart (1983) Primary structure of the oligosaccharide determinant of blood group Cad specificity. J. Biol. Chem. 258: 7691-7695.

Blanchard, D., F. Piller, B. Gillard, D. Marcus, and J.-P. Cartron (1985) Identification of a novel ganglioside on erythrocytes with blood group Cad specificity. J. Biol. Chem. 13: 7813-7816.

Carrino, D. A., and A. I. Caplan (1982) Isolation and characterization 
of proteoglycans synthesized by skeletal muscle. J. Biol. Chem. 257: 14145-14154.

Cartron, J.-P., and A. T. Nurden (1979) Galactosyltransferase and membrane glycoprotein abnormality in human platelets from $\mathrm{Tn}$ syndrome donors. Nature 282: 621-623.

Ceri, H., D. Kobiler, and S. H. Barondes (1981) Heparin-inhibitable lectin: Purification from chicken liver and embryonic chicken muscle. J. Biol. Chem. 256: 390-394.

Chien, J.-C., and E. L. Hogan (1983) Novel pentahexosyl ganglioside of the globoseries purified from chicken muscle. J. Biol. Chem. 258: 10727-10730.

Clausen, H., J. B. Levery, E. Nudelman, S. Tsuchiya, and S.-I. Hakomori (1985) Repetitive A epitope (type 3 chain A) defined by blood group $\mathrm{A}_{1}$-specific monoclonal antibody $\mathrm{TH}-1$ : Chemical basis of qualitative $A_{1}$ and $A_{2}$ distinction. Proc. Natl. Acad. Sci. USA 82: 1199-1203.

Couchman, J. R., B. Caterson, J. E. Christner, and J. R. Baker (1984) Mapping by monoclonal antibody detection of glycosamino-glycans in connective tissues. Nature 307: 600-652.

Covault, J., and J. R. Sanes (1986) Distribution of N-CAM in synaptic and extrasynaptic portions of developing and adult skeletal muscle. J. Cell Biol. 102: 716-730.

Cummings, R. D., and S. Kornfeld (1982) Characterization of the structural determinants required for the high affinity interaction of asparagine-linked oligosaccharides with immobilized Phaseolus vulgaris leukoagglutinating and erythroagglutinating lectins. J. Biol. Chem. 257: 11230-11234.

Danishefsky, I., H. B. Eiber, and J. J. Carr (1960) Investigations on the chemistry of heparin. I. Desulfation and acetylation. Arch. Biochem. Biophys. 90: 114-121.

Debray, H., D. Decout, G. Strecker, G. Spik, and J. Montreuil (1981) Specificity of twelve lectins towards oligosaccharides and glycopeptides related to $N$-glycosylproteins. Eur. J. Biochem. 117: 41-45.

Doctor, B. P., S. Camp, M. K. Gentry, S. S. Taylor, and P. Taylor (1983) Antigenic and structural differences in the catalytic subunits of the molecular forms of acctylcholincstcrasc. Proc. Natl. Acad. Sci. USA 80: 5717-5771.

Dodd, J., D. Solter, and T. M. Jessel (1984) Monoclonal antibodies against carbohydrate differentiation antigens identify subsets of primary sensory neurones. Nature 311: 469-472.

Donald, A. S. R., C. R. C. Soh, W. M. Watkins, and W. T. J. Morgan (1982) $N$-acetyl-D-galactosaminyl- $\beta$-(1-4)-D-galactose: A terminal non-reducing structure in human blood group $\mathrm{Sd}^{\mathrm{a}}$-active TammHorsfall urinary glycoprotein. Biochem. Biophys. Res. Commun. 104: $58-65$.

Ellman, G. L., K. D. Courtney, V. Andres, Jr., and R. M. Featherstone (1961) A new and rapid colorimetric determination of acetylcholinesterase activity. Biochem. Pharmacol. 7: 88-95.

Etzler, M. E., and E. A. Kabat (1970) Purification and characterization of a lectin (plant hemagglutinin) with blood group A specificity from Dolichos biflorus. Biochemistry 9: 869-877.

Etzler, M. E., S. Gupta, and C. Borrebaeck (1981) Carbohydrate binding properties of the Dolichos biflorus lectin and its subunits. J. Biol. Chem. 256: 2367-2370.

Furukawa, K., H. Clausen, S.-I. Hakomori, J. Sakamoto, K. Look, A. Lundblad, M. J. Mattes, and K. O. Lloyd (1985) Analysis of the specificity of five murine anti-blood group A monoclonal antibodies, including one that identifies type 3 and type $4 \mathrm{~A}$ determinants. Biochemistry $24: 7820-7826$.

Hakamori, S.-I. (1981) Glycosphingolipids in cellular interaction, differentiation and oncogenesis. Annu. Rev. Biochem. 50: 733-764.

Hall, Z. W. (1973) Multiple forms of acetylcholinesterase and their distribution in endplate and non-endplate regions of rat diaphragm muscle. J. Ncurobiol. 4: 343-361.

Hammerstrom, S., L. A. Murphy, I. J. Goldstein, and M. E. Etzler (1977) Carbohydrate binding specificity of four $N$-acetyl-D-galactosamine "specific" lectins: Helix pomatia A hemagglutinin, soy bean agglutinin, lima bean lectin, and Dolichos biflorus lectin. Biochemistry 16: $2750-2755$.

Hansen-Smith, F. M. (1984) Relationship between junctional acetylcholine receptor clustering and Dolichos biflorus agglutinin receptors in regenerating skeletal muscle. Soc. Neurosci. Abstr. 10: 924.

Hirohashi, S., H. Clausen, T. Yamada, Y. Shimosato, and S.-I. Hakomori (1985) Blood group A cross-reacting epitope defined by monoclonal antibodies NCC-LU-35 and -81 expressed in cancer of blood group O or B individuals: Its identification as Tn antigen. Proc. Natl. Acad. Sci. USA 82: 7039-7043.
Holthoffer, H. (1983) Lectin binding sites in kidney. A comparative study of 14 animal species. J. Histochem. Cytochem. 31: 531-537.

Hounsell, E. F., and T. Feizi (1982) Gastrointestinal mucins. Med. Biol. 60: 227-236

Johnson, C. D., and R. L. Russell (1975) A rapid, simple radiometric assay for cholinesterase, suitable for multiple determinations. Anal. Biochem. 64: 229-238.

Kannagi, R., S. B. Levery, F. Ishigami, S.-I. Hakamori, L. H. Shevinsky, B. B. Knowles, and D. Solter (1983a) New globoseries glycosphingolipids in human terato carcinoma reactive with the monoclonal antibody directed against a developmentally regulated antigen, SSEA 3. J. Biol. Chem. 258: 8934-8942.

Kannagi, R., N. A. Cochran, F. Ishigami, S.-I. Hakamori, P. W. Andrews, B. B. Knowles, and D. Solter (1983b) Stage-specific embryonic antigens (SSEA-3 \& -4) are epitopes of a unique globoseries ganglioside isolated from human teratocarcinoma cells. EMBO J. 2: 2355-2361.

Kaupmann, K., P. Heimann, and H. Jockusch (1985) N-Acetyl-galactosamin specific lectin binding in mammalian muscle: Dependence on development, mutations and species. Eur. J. Cell Biol. (Suppl. 12) 39: 19.

Kobata, A. (1984) The carbohydrates of glycoproteins. In The Biology of Glycoproteins, R. J. Ivatt, ed., pp. 183-234, Plenum, New York.

Lee, S. L., S. Heinemann, and P. Taylor (1982) Structural characterization of the asymmetric $(17+13) \mathrm{S}$ forms of AChE from Torpedo. I. Analysis of subunit composition. J. Biol. Chem. 257: 12283-12291.

Lis, H., and N. Sharon (1986) Lectins as molecules and tools. Annu. Rev. Biochem. 55: 35-87.

Mansson, J.-E., and S. Olofsson (1983) Binding specificities of the lectins from Helix pomatia, soybean and peanut against different glycosphingolipids in liposome membrane. FEBS Lett. 156: 249-252.

Marchase, R. B. (1987) Glucose-1-phosphate containing glycoproteins and interneuronal adhesion. Am. Zool. 27: 179-187.

Margolis, R. V., and R. K. Margolis (1978) Complex Carbohydrates of Nervous Tissue, Plenum, New York.

Massoulie, J., and S. Bon (1982) The molecular forms of cholinesterase and acetylcholinesterase in vertebrates. Annu. Rev. Neurosci. 5: 57106.

McMahan, U. J., J. R. Sanes, and L. M. Marshall (1978) Cholinesterase is associated with the basal lamina at the neuromuscular junction. Nature 271: 172-174.

Mohan, P. S., and R. G. Spiro (1986) Macromolecular organization of basement membranes. Characterization and comparison of glomerular basement membrane and lens capsule components by immunochemical and lectin affinity procedures. J. Biol. Chem. 261: $4328-4336$

Natoli, E. J., Jr., P. O. Livingston, C. S. Pukel, K. O. Lloyd, H. Wiegandt, J. Szalay, H. F. Oettgen, and L. J. Old (1986) A murine monoclonal antibody detecting $N$-acetyl-and $N$-glycolyl-G $\mathrm{G}_{\mathrm{M} 2}$. Characterization of cell surface reactivity. Cancer Res. 46: 4116-4120.

Obata, K., M. Oide, and S. Handa (1977) Effects of glycolipids on in vitro development of neuromuscular junction. Nature 266: 369-371.

Olden, K., J. B. Parent, and S. L. White (1982) Carbohydrate moieties of glycoproteins: A reevaluation of their function. Biochem. Biophys. Acta 650: 209-232.

Pereira, M. E. A., and E. A. Kabat (1974) Immunochemical studies on the specificity of soybean agglutinin. Carbohydrate Res. 37: 89102.

Piller, V., F. Piller, and J.-P. Cartron (1986) Isolation and characterization of an $\mathrm{N}$-acetylgalactosamine-specific lectin from salvia sclerea seeds. J. Biol. Chem. 261: 14069-14075.

Ravdin, P., and D. Axelrod (1977) Fluorescent tetramethyl rhodamine derivatives of $\alpha$-bungarotoxin: Preparation, separation, and characterization. Anal. Biochem. 80: 585-592.

Ribera, J., J. E. Esquerda, J. X. Comella, M. A. Poca, and M. J. Bellmunt (1987) Receptors to agglutinin from Dolichus biflorus (DBA) at the synaptic basal lamina of rat neuromuscular junction. Cell Tissue Res. 248: 111-117.

Roberts, D. D., and I. J. Goldstein (1984) Effect of carbohydrate and metal ion binding on the reactivity of the essential thiol groups of lima bean lectin. J. Biol. Chem. 259: 903-908.

Rotundo, R. L. (1984) Asymmetric AChE is assembled in the Golgi apparatus. Proc. Natl. Acad. Sci. USA 81: 479-483.

Sahagian, G. G. (1987) The mannose-6-phosphate receptor and its role in lysozomal enzyme transport. In Vertebrate Lectins, K. Olden and J. B. Parent, eds., pp. 46-64, Van Nostrand Reinhold, New York. 
Sanes, J. R., and J. M. Cheney (1982) Lectin binding reveals a synapsespecific carbohydrate in skeletal muscle. Nature 300: 646-647.

Sanes, J. R., and Z. W. Hall (1979) Antibodies that bind specifically to synaptic sites on muscle fiber basal lamina. J. Cell Biol. 83: 357370.

Scott, L. J., and J. R. Sanes (1984) A lectin that selectively stains neuromuscular junctions binds to collagen-tailed AChE. Soc. Neurosci. Abstr. 10: 546 .

Scott, L. J., and J. R. Sanes (1987) A synapse-specific carbohydrate at the neuromuscular junction is associated with both acetylcholinesterase and a glycolipid. Soc. Neurosci. Abstr. 13: 1636.

Shevinsky, L. H., B. B. Knowles, I. Damjanov, and D. Solter (1982) Monoclonal antibody to murine embryos defines a stage-specific embryonic antigen expressed on mouse embryo and human teratocarcinoma cells. Cell 30: 697-705.

Smith, C. E., and C.-P. Ko (1986) A plant lectin, peanut agglutinin, binds specifically at the neuromuscular junction of the frog. Soc. Neurosci. Abstr. 12: 1500.

Stern, P. L., K R Willison, E. Lennox, G. Galfre, C. Milsteen, D. Secher, D. Zieger, and T. Springer (1978) Monoclonal antibodies as probes for differentiation and tumor-associated antigens: A Forssman spccificity in teratocarcinoma stem cells. Cell 14: 775-783.
Storer, T. I., R.L. Usinger, R. C. Stebbins, and J. W. Nybakken (1972) General Zoology, McGraw-Hill, New York.

Svennerholm, L., A. Bruce, J.-E. Mansson, B.-M. Rynmark, and M.T. Vanier (1972) Sphingolipids of human skeletal muscle. Biochim. Biophys. Acta 280: 626-636.

Tippett, R., P. W. Andrews, B. B. Knowles, D. Solter, and P. N. Goodfellow (1986) Red cell antigens P (globoside) and Luke: Identification by monoclonal antibodies defining the murine stage-specific embryonic antigens -3 and -4 (SSEA-3 and SSEA-4). Vox Sang. 51: $53-$ 56.

Tollefsen, S., and S. Kornfeld (1983) The $B_{4}$ lectin from vicia villosa seeds interacts with GalNAc residues $\alpha$-linked to serine or threonine residues in cell surface glycoproteins. J. Biol. Chem. 258:5172-5176.

Tollefsen, S., and S. Kornfeld (1984) The $B_{4}$ lectin from vicia villosa seeds interacts with $\mathrm{N}$-acetylgalactosamine residues on erythrocytes with blood group Cad specificity. Biochem. Biophys. Res. Commun. 123: 1099-1106.

Younkin, S. E., C. Rosenstein, P. L. Collins, and T.L. Rosenberry (1982) Cellular localization of the molecular forms of acetylcholinesterase in rat diaphragm. J. Biol. Chem. 257: 13630-13637. 